Digital Press Social Sciences and Humanities

Les étapes de traduire des proverbes français en indonésien

Ismirani Mardalena

Proceeding of Conférence internationale sur le français 2018

Joesana Tjahjani, Merry Andriani, Sajarwa, Wening Udasmoro (eds) 


\title{
Les étapes de traduire des proverbes français en indonésien
}

\author{
Ismirani Mardalena \\ Universitas Indonesia, Depok, Indonesia \\ e-mail : ismiranimardalena@gmail.com
}

\section{Résumé}

La traduction des proverbes n'est pas une activité simple. Les traducteurs font face toujours aux problèmes. Ils doivent transférer les messages des proverbes pour que la bonne traduction soit faite sans changer ou éliminer les principes des proverbes étant les morales et les cultures. Cette traduction ne se pose pas seulement aux problèmes d'une inexistence de sens et formes du proverbe indonésien, mais aussi ceux du type des proverbes. À partir de ces problèmes-là, l'auteur a analysé les problèmes trouvés pour découvrir les bonnes façons de traduire les proverbes français en indonésien. Les proverbes analysés viennent d'une fable française très connu, "Les Fables de La Fontaine" datant 1668 au 1678. Quatorze titres de fables ont été choisis et il y a seize proverbes trouvés. En utilisant la théorie de traduction de Nida et Taber développée par Hoed et la stratégie paraphrase de Mona Baker, les proverbes ont été traduits et analysés. Cette recherche nous donne des étapes de traduire des proverbes français en indonésien. Il y a trois éléments pertinents que les traducteurs doivent prendre attention en traduisant des proverbes pour que la bonne traduction soit achevée. Le premier élément c'est qu'il faut trouver les mots clés, deux mots au maximum et que ce soit un mot ou un syntagme ou un synthème. Il faut aussi faire attention à choisir des proverbes indonésiens car ils sont des fois différentes que ceux de langue source. À la fin, il faut aussi garder la cohérence du texte traduit.

\section{Mots-clés}

traduction, proverbe, français-indonésien

\begin{abstract}
The translation of proverbs is not a simple activity. Translators still face problems. They must transfer the message so that the right translation will be done without change or eliminate the principles of the proverbs being moral and culture. This translation arises not only at the problems of a lack of direction and forms of the Indonesian proverb, but also those of the type of proverbs. From these problems, we analyzed the problems to discover good ways to translate. The data is collected from a well-known French fable, "Les Fables de La Fontaine" from 1668 to the 1678. Fourteen titles of fables have been chosen and sixteen proverbs were founded. Using Nida and Taber's theory of translation that is developed by Hoed and Mona Baker's paraphrase strategy, proverbs were translated and analyzed. This research gives us steps to translate French proverbs in Indonesian. There are three relevant elements that translators must take into account. Firstly, is to find key words, two words maximum, whether a word or a phrase. Secondly, be careful to choose Indonesian proverbs as they are sometimes different than those of source language. And thirdly, we must also keep the consistency of the translated text.
\end{abstract}

\section{Keywords}

translation, proverb, French-Indonesian 


\section{Introduction}

\subsection{L'arrière-plan}

Aujourd'hui, on trouve beaucoup de littératures françaises étant traduites en indonésien, surtout la littérature pour les enfants, par exemple L'histoire de 10 Chevaliers traduite par Ismirani Mardalena, La Petite Imagerie : Les Volcans ; La Petite Imagerie : Le Zoo traduites par Airin Miranda, et Émile et Arthur, traduite par Diah Kartini Lasman. Ces phénomènes-là nous montrent que la demande de la traduction d'une littérature française est de plus en plus élevée. Cette forte demande doit être en ligne au développement des compétences de traducteurs. Les traducteurs sont contestés d'être de plus en plus mieux pour qu'ils puissent faire face a celui étant l'un de défis mondiaux.

Cette recherche a pour l'objectif de donner aux traducteurs des nouveaux connaissances sur la traduction des expressions idiomatiques étant toujours trouvés quand ils font des traductions de la littérature. Ils rencontrent souvent les expressions figés ou idiomatiques qui se posent toujours des problèmes. L'un de ceux est le proverbe.

L'utilisation des proverbes dans la littérature existe depuis des siècles derniers. Whiting (1932) sur Honneck (1997, p. 11) a noté que le proverbe a un rôle pertinent dans la littérature grecque. Les philosophes célèbres comme Socrates, Plato, et Aristoteles l'avait utilisé dans leurs œuvres. En Europe, surtout en France, l'apparition de proverbes dans la littérature avait commencé depuis l'Antiquité et il a eu un grand succès au Moyen-âge (XI-XV siècles) et au siècle de Classique (XVI-XVIII siècle).

$\mathrm{Au}$ XVII siècles, le classicisme et le baroque ont caractérisé cette époque. Les œuvres plus célèbres sont ceux de Molière et Jean de La Fontaine. Molière utilise le proverbe pour configurer son populisme dans son script, par exemple "qui veut noyer son chien l'accuse de la rage" (Les femmes savantes, acte II, scène V). Et Jean de La Fontaine (1621-1695) a fait dans ses Fables (1668-1693) une critique sociale et politique de la société française à travers le monde animal. Les formes sentencieuses, "ventre affamé n'a point d'oreilles" (Livre 9o, fable XVIII : Le Milan et le Rossignol), aident à la fable à atteindre ses objectifs principaux : apprendre et moraliser.

Compte tenu de l'importance de la fonction et de l'utilisation des proverbes dans les œuvres littéraires, cet auteur discute des étapes de la traduction des proverbes français en indonésien.

\subsection{Le contexte}

Cet auteur a choisi quatorze fables de Jean de La Fontaine (1968 et 1978) comme sources de recherche car ces fables contiennent des proverbes qui jouent un rôle important dans chaque histoire. L'utilisation de proverbes dans chaque fable est le style de l'auteur pour raconter une histoire.

De ces sources, cet auteur a trouvé les proverbes français traduits par deux formes : celle du proverbe indonésien et celle d'une paraphrase. Exemple :

Celle du proverbe

La source : (1) Les petits ont pâti des sottises des grands.

(2) On a souvent besoin d'un plus petit que soi.

(3) L'avarice perd tout en voulant tout gagner.

La cible $\quad$ : (1) Gajah berjuang sama gajah, pelanduk mati di tengah-tengah.

(2) Harimau putung karena penjara, pelanduk kecil menolakkan.

(3) Harapkan burung terbang tinggi, punai di tangan dilepaskan

Celle d'une paraphrase

La source : (1) Patience et longueur de temps Font plus que force ni que rage.

(2) Chacun à son métier doit toujours s'attacher.

(3) Garde-toi, tant que tu vivras, de juger des gens sur la mine.

La cible : (1) Kesabaran dan waktu yang panjang lebih baik daripada kekuatan dan kemarahan.

(2) Setiap orang harus fokus pada keahliannya.

(3) Ingatlah selama hidupmu, jangan menilai seseorang dari tampilan

\subsection{La problématique}

Ces explications-là ont incité cet auteur de penser a un problème : quels sont les facteurs pertinents dans la traduction du proverbe français en indonésien? 


\subsection{Les objectifs}

L'objectif de cette recherche est pour expliquer les facteurs influencés aux étapes de traduire les proverbes français en indonésiens alors que ceux soient utiles pour les traducteurs indonésiens, surtout les traducteurs de littératures et les étudiants qui étudient la traduction.

\subsection{Les théories pertinentes}

\subsubsection{Les proverbes}

Mieder (1993, p. 5; p. 24) définit un proverbe comme « des phrases courtes qui existent dans une société qui contient un élément de la sagesse, la vérité, la morale, et les vues traditionnelles sous forme de métaphores, de forme formelle, et toujours souvenu et transmis d'une génération à l'autre ». Norrick (1985, p. 40) fournit une définition des proverbes comme ...a traditional, conversational, didactic genre with general meaning, a potential free conversational turn, preferably with figurative meaning. (Un genre traditionnel, conversation didactique au sens générale, a un potentiel d'alterner librement dans la conversation et contient généralement une signification figurative).

Ainsi, nous pouvons conclure que le proverbe est des expressions idiomatiques dont l'utilisation est fixée, sous forme de structure figée et concise. En plus, il a un sens didactique et grand, il peut aussi avoir un sens figuré, et il contient un élément de la sagesse, la vérité et la morale. La raison pourquoi les proverbes sont très utilisés par les gens est parce qu'il y a du processus de transfert des cultures de génération en génération.

\subsubsection{Caractéristiques et structure du proverbe}

Selon Mieder (2004), le proverbe est un type de discours ou d'expression traditionnelle ou encore appelé texte traditionnel car il montre les caractéristiques des textes traditionnels. Celles-là sont :

1. la structure est figée ; ce qui signifie que l'ordre entre les éléments ne peut pas être muté, et parmi les éléments ne peuvent pas être insérés des mots ou d'autres éléments.

2. les mots qui remplissent le texte ne peuvent pas être remplacés par d'autres mots ; si le mot est remplacé par un autre mot, il aura un sens différent.

3. Le texte doit être considéré comme une unité. C'est-à-dire qu'un élément ne peut pas recevoir une explication ajoutée. Par exemple le proverbe La vie est dur, si on leur donne l'explication -très- il devient La vie est très dur, l'identité de ce discours a vraiment changé.

Silverman-Weinreich (1978) dans Honeck (1997, pp. 13-14) spécifie les caractéristiques des proverbes basées sur un point linguistique. Selon lui, les proverbes sont une unité entre phonologie, syntaxe et sémantique. Proverbe peut être des phrases structurées, des clauses (de construction prédicative), ou la peine, que ce soit la peine de structure simple ou une phrase composée et phrases impératives peut être construit, que ce soit impératif impérieusement positif et négatif, interrogative, comparatif, paraboles. Le proverbe comporte au moins deux caractères grammaticaux (il y a un sujet concret ou abstrait et il y a une nominalisation de verbes ou pas), un marqueur de sémantique (métaphore, paradoxe, ou hyperbole), et un marqueur phonologique (une rime, une assonance, une consonance ou une allitération).

\subsubsection{Type de proverbe}

Selon Bhuvaneswar (2000, pp. 2-5), il existe deux types de proverbes :

1. Proverbe littéral.

Ce proverbe est un proverbe qui a un sens qui correspond au sens référentiel de ses éléments constitutifs. Par exemple le proverbe français :

«L'avarice perd tout en voulant tout gagner ». Ce proverbe existe dans une histoire qui raconte une histoire de quelqu'un qui n'a jamais été satisfait de ce qu'il a, qui en veut toujours plus. Le message est représenté par le mot l'avarice qui a le sens 'quelqu'un qui représente un rapport de l'avare' et perd qui a le sens 'ne plus avoir'. Sur la base des mots qui le représentent, on peut voir que le sens référentiel du proverbe est directement adressé au message à délivrer. Ainsi, le sens du proverbe est le même que le sens référentiel de l'énoncé du proverbe.

2. Proverbe figuratif.

Ce proverbe utilise les styles de langues figuratifs, tels que la comparaison, l'hyperbole, le paradoxe, la métaphore, de sorte que le sens de référence dépend de la signification figurative utilisée dans le proverbe, par exemple le proverbe indonésien : Tong kosong nyaring bunyinya (Le 
cuve vide sonne fortement). Ce proverbe est utilisé dans la situation où il y a quelqu'un qui parle beaucoup, mais ça ne dit rien. Le message est représenté par un syntagme tong kosong (le cuve vide) et le verbe nyaring (sonne fortement). Ce proverbe utilise la métaphore. il a métaphorisé le sens du " cuve vide » avec les «humains». Le syntagme tong kosong est choisie parce que les conditions sont les mêmes entre les cuves vides et les humains, c'est-à-dire que les cuves vides sonnent plus fortes que les cuves contenant quelque chose. La condition est assimilée aux humains qui ne sont pas bien informés, mais ils parlent plus que les humains avec des bonnes connaissances. Ainsi, le sens du proverbe est le sens référentiel qui vient du sens des styles de langues figuratifs.

Le type de proverbe est déterminé à partir des mots-clés utilisés. Chaque proverbe a au moins deux mots-clés. Le proverbe littéral utilise des mots-clés qui sont directement centrés sur le message, ce qui se traduit par la même signification référentielle que l'énoncé et le sens du proverbe. Le proverbe figuratif utilise des mots-clés qui ne sont pas directement axés sur ses messages, mais utilisent plutôt la signification des mots-clés qui font partie de l'assemblage, de sorte que le sens du proverbe dépend de la signification des figures de styles utilisés.

\subsubsection{La procédure de traduction}

Selon Hoed (2006, p. 11), les traducteurs rencontrent deux problèmes pratiques en traduction. Tout d'abord, le traducteur ne comprend pas la signification du mot, de la phrase ou du paragraphe, de sorte qu'il ne comprend pas bien le message. Deuxièmement, le traducteur avait de la difficulté à traduire même s'il comprenait le texte source. Pour surmonter cet obstacle, les traducteurs doivent appliquer la procédure de traduction qui, selon Nida et Taber (1974, p. 12), composé de traduction en trois étapes : l'analyse (comprendre la source), le transfert (transférer le message dans la tête), et la restructuration (exprimer des messages dans la langue cible).

Pour soutenir ces trois étapes-là, Hoed (2006) a ajouté quatre niveaux de traduction. Le premier et deuxième niveau concernant le texte source, quant à eux, le troisième et quatrième niveau concernant le texte cible. Ceux sont: (1) le niveau textuel, c'est quand le traducteur essaie de comprendre le texte à traduire principalement au niveau des mots et des phrases; (2) le niveau de référentiel, c'est quand le traducteur va au dehors du texte pour savoir à quoi est référé d'un mot, d'un terme ou d'une expression dans le texte; (3) le niveau de cohésion, qui examine la cohésion du texte de traduction; (4) le niveau d'acceptance, qui consiste à vérifier l'acceptabilité du lecteur cible.

Dans cette recherche, cet auteur utilise ces niveaux-là, sauf celui de numéro deux, pour analyser et trouver les facteurs importants dans la traduction de proverbe. Le deuxième niveau n'est pas utilisé car il est plus approprié pour connaître les éléments culturels contenus dans le proverbe.

\subsubsection{La stratégie de paraphrase}

Selon Baker (2011, p. 67-86), cette stratégie est la stratégie la plus fréquemment utilisée pour traduire des expressions idiomatiques. Les traducteurs sont fondamentalement difficiles à trouver des idiomes de la cible qui sont équivalent avec celle de la source ou ils font face au problème de l'inexistence des idiomes de la cible. Faire de la paraphrase devient donc une stratégie plus rapide et plus facile.

Exemple :

La source : : Aide-toi, le ciel t'aidera

La cible : Tuhan akan menolong orang yang berusaha

(Dieu aidera ceux qui essaient)

\section{Méthode}

Les sources du contexte viennent de quatorze fables de Jean de La Fontaine et de leurs traductions en indonésien. Seize proverbes français se traduisent par sept proverbes indonésiens et neuf paraphrases. Cette recherche s'est déroulée en deux étapes. Tout d'abord, classer les proverbes par type de proverbe en utilisant la théorie de Bhuvaneswar. La deuxième, les analyser à quatre niveaux de Benny pour découvrir les facteurs importants dans la traduction des proverbes.

\section{Résultats et discussion}

Seize proverbes de la langue source consistent en quinze proverbes littéraux et un proverbe figuratif. Ceux-ci de littéraux traduits par six proverbes figuratifs de la langue cible et neuf paraphrases. Et celui-là de figuratif traduit par le proverbe figuratif de la langue cible. 
Le principal problème dans la traduction des proverbes français est l'absence de proverbes de la langue cible qui sont équivalents de celle de la langue source. Cela est dû aux différents types de proverbes entre les proverbes de la source et de la cible. Le proverbe de la langue source (le français) est un proverbe littéraire, tandis que celui de la cible (l'indonésien) est un proverbe figuratif. Les traducteurs donc choisissent des stratégies de paraphrase pour résoudre ce problème. Un autre problème est le problème de la cohérence entre le proverbe de la langue cible et le texte de la langue source. La cause du problème est la différence entre le mot-clé de proverbes de langue sources et ceux-là de la cible. Dans ce cas, le traducteur retourne aux lecteurs cibles et au but de la traduction pour que la traduction soit acceptée par le lecteur cible.

Il y a trois choses importantes auxquelles le traducteur doit faire attention dans la traduction des proverbes. La première chose principale est le mot-clé du proverbe de la langue source, la deuxième est la sélection du proverbe de la langue cible, et la troisième est la cohésion entre le proverbe de cible et le texte source. Ceux-ci sont les exemples comment les proverbes sont analysés.

\subsection{La traduction est le proverbe de la langue cible}

Tableu 1 La traduction est le proverbe de la langue cible

\begin{tabular}{|l|l|}
\hline \multicolumn{1}{|c|}{ La Langue Source } & \multicolumn{1}{c|}{ La Langue Cible } \\
\hline Les petits ont pâti des sottises des grands. & $\begin{array}{l}\text { Gajah berjuang sama gajah, pelanduk mati di } \\
\text { tengah-tengah. }\end{array}$ \\
& $\begin{array}{l}\text { (Les éléphants se battent avec les éléphants, } \\
\text { Les cornes mortes au milieu) }\end{array}$ \\
\hline
\end{tabular}

Ce proverbe se trouve dans une fable qui raconte les grenouilles, victimes de deux batailles de buffles qui tentent de gagner une femelle buffle. Selon le contexte de l'histoire, la signification du proverbe est qu'une personne petite ou plus faible est souvent victime de la stupidité d'une grande personne.

Le proverbe Les petits ont pâti des sottises des grands traduit par un proverbe de cible Gajah berjuang sama gajah, pelanduk mati di tengah-tengah car ils ont des significations similaires. Les explications sont cidessous.

La première, au niveau textuel, le mot clé du proverbe est le mot les petits qui a la signification « qui a peu de valeur » et de « pâtir » qui signifie « souffrir » et des « grands » qui signifie « qui une importance sociale ou politique ». Selon la base des mots clés ci-dessus, la signification du proverbe de la langue source est une petite personne qui souffrira du comportement insensé des grandes personnes.

La signification du proverbe de la langue source est similaire à celui de la langue cible Gajah berjuang sama gajah, pelanduk mati di tengah-tengah. Ce proverbe signifie d'une personne formidable ou des dirigeants qui se sont disputés, mais le petit peuple qui souffre en dessous de ceux. On dit que c'est similaire parce que le proverbe de langue source et celui de langue cible contiennent la signification de "petit peuple » et de " souffrance » bien que les mots-clés utilisés sont différents. La signification de « petit peuple » dans le proverbe de langue source est représenté littéralement par le nom les petits et le sens de « souffrance » par le verbe pâtir (souffrance). En attendant que sur le proverbe de langue cible, il utilise une figure de style, dans ce cas est la métaphore, pelanduk (des cornes) pour «petit peuple » et le parabole pour le sens de « souffrance » par des phrases mati di tengah-tengah (mortes au milieu).

La deuxième, au niveau de la cohésion, aucun problème ne se pose car le mot utilisé dans le proverbe de langue cible est cohérent dans le contexte de l'histoire, comme pelanduk ayant un lien avec les petits parce qu'ils ont la signification petite et faible. Bien que le type de proverbe de langue source soit différent à celui de langue cible, il est un proverbe littéral, tandis que celui de langue cible est un proverbe figuratif, le proverbe de langue cible est cohérent à celui de la source. La signification du proverbe de langue cible est le sens figuré de la phrase qui compare les querelles entre des personnes qui ont du pouvoir et qui causent la souffrance des petits comme des éléphants qui combattent des éléphants, mais la corne morte au milieu.

La troisième, au niveau d'acceptance, le proverbe de langue cible a une structure de phrase traduite utilisant la méthode de traduction idiomatique et la stratégie idiomatique. La forme de la traduction est un proverbe de langue cible. Cette forme de traduction est raisonnable et acceptable pour le lecteur cible car la signification est similaire entre le proverbe de langue source et celui de langue cible. 


\subsection{La traduction est la paraphrase}

Tableu 2 La traduction est la paraphrase

\begin{tabular}{|c|c|}
\hline La Langue Source & La Langue Cible \\
\hline Chacun à son métier doit toujours s'attacher. & Setiap orang harus fokus pada keahliannya. \\
& \\
\hline
\end{tabular}

Ce proverbe se trouve dans des fables qui racontent un loup qui prétend être un médecin pour qu'il puisse manger un cheval qui est seul dans une prairie. Selon le contexte de l'histoire, le message de ce proverbe est que tout le monde doit se concentrer bien à ses capacités.

La première, au niveau textuel, le mot clé de ce proverbe sont le métier qui signifie 'occupation permanente qui possède certains caractères du métier' et s'attacher qui signifie 'être attacher'. Selon ces mots-clés, la signification de ce proverbe est que tout le monde doit se concentrer sur les capacités dont ils ont au travail.

La deuxième, au niveau de la cohésion, aucun problème ne se pose car la traduction Setiap orang memiliki kemampuan dalam mengerjakan sesuatu; tetaplah pada kemampuan itu est cohérent. Le mot kemampuan (les capacités) et une phrase menyembuhkan penyakit ont un lien au mot le métier de la langue source.

La troisième, au niveau d'acceptance, le proverbe de langue cible a une structure de phrase traduite utilisant la méthode de traduction communicative et la stratégie paraphrase. La forme de la traduction est une paraphrase de la langue cible. Cette forme de traduction est raisonnable et acceptable pour le lecteur cible car le mot kemampuan signifie "capacité, compétence" et c'est universellement applicable. De plus, le contexte du texte source et celui de cible indiquent les capacités qui sont mal utilisées, de sorte que la sélection des mots de capacité en tant que mot-clé dans le texte cible est appropriée au contexte.

\section{Conclusion}

Il y a quelques étapes à prendre en compte pour traduire les proverbes français en indonésiens. (1) La première est les traducteurs doivent d'abord trouver au moins deux mots clés du proverbe de la source. Les mots-clés peuvent être sous forme de mots ou de syntagme ou de syntème. À partir de ces mots-clés, les traducteurs peuvent connaître le thème de ce proverbe. (2) Ensuite, selon la base de ce thème, les traducteurs peuvent rechercher des équivalents de ce proverbe dans la cible sur une page Internet. La recherche sur la page Internet est fait pour plusieurs raisons : (a) les traducteurs ne peuvent pas chercher les proverbes en regardant le thème dans le dictionnaire de proverbe indonésien car celui est basés sur l'ordre alphabétique, (b) les traducteurs peuvent obtenir rapidement le proverbe avec le thème désiré plus simplement en tapant le thème dans la colonne de recherche, par exemple le thème est la cupidité, alors on tape "proverbe indonésien de la cupidité". Cette recherche vise à obtenir le proverbe de la cible avec le même thème de celui de la source.

La troisième (3) est les traducteurs doivent être capable de choisir le bon proverbe équivalent. Ça veut dire le proverbe de la cible doit avoir le même objectif que celui de la source. (4) Il faut consulter au dictionnaire de proverbe indonésien pour savoir si celui est correct ou pas. Une fois que le traducteur a trouvé les proverbes de la cible au même thème sur la page Internet, le traducteur doit vérifier un à un la signification du proverbe trouvé au dictionnaire de proverbe indonésien.

La dernière étape est (5) les traducteurs doivent faire attention à la cohérence entre le proverbe de la cible et le texte source. Bien que le proverbe a une caractéristique, celui est indépendante, qui peut se suffire à lui-même, ça signifie que ce proverbe peut être comprise soit par le contexte ou non, mais les proverbes dans cette recherche font partie d'une histoire de texte sous la forme d'une fable. Alors, les proverbes de la cible doivent être cohérents avec le contexte de l'histoire. Quand les traducteurs trouvent un proverbe cible qui semble incohérent, il faut retourner au genre de proverbes et les ré-analyser pour faire en sorte que le proverbe est cohérent. S'il n'est pas encore cohérent, les traducteurs peuvent utiliser la stratégie paraphrase. 


\section{Références}

Baker, M. (2011). In Other Words: A coursebook on translation, second edition. New York: Routledge.

Bhuvaneswar, C. (2000). Derivation of Meaning in Proverbs: A Karmik Linguistic Analysis (No. 5).

Hyderabad.

Hoed, B. H. (2006). Penerjemahan dan Kebudayaan. Bandung: Dunia Pustaka Jaya.

Honneck, R. P. (1997). A Proverb in Mind: The Cognitive Science of Proverbial Wit and Wisdom. Mahwah: Lawrence Erlbrum Associates.

Mieder, W. (2004). Proverbs: A handbook. New York: Greenwood Publishing Group.

Mieder, W., \& Dundes, A. (1981). The Wisdom of Many: Essays on the Proverb. New York: Garland Pub.

Nida, E. A., \& Taber, C. R. (1974). The Theory and Practice of Translation. Leiden: Brill. https://doi.org/10.2753/RSL1061-1975010389

Norrick, N. R. (1985). How Proverbs Mean: Semantic Studies in English Proverbs. New York: Mouton. https://doi.org/10.2307/415429 